\title{
LA CATEGORÍA "INFANCIA ANORMAL" EN LA CONSTRUCCIÓN DE UNA TAXONOMÍA SOCIAL EN EL PRIMER TERCIO DEL SIGLO XX"
}

\author{
Rafael Huertas y Mercedes del Cura
}

Dpto. de Historia de la Ciencia - Centro de Estudios Históricos - CSIC. Madrid

\section{RESUMEN}

El presente trabajo pretende estudiar algunos de los principios básicos que intervinieron en $\mathrm{cl}$ cambio de paradigma que sobre la deficiencia mental se produjo en las primeras décadas del siglo XX. Tomando como fuentes principales las aportaciones de Binet y Simon en Francia y de Sante de Sanctis en Italia, se analiza la construcción de la categoría de "infancia anormal", relacionándola con el nuevo y privilegiado espacio de observación que supuso la Escuela Obligatoria.

Se concluye con una reflexión sobre la falaz utilización de la "inteligencia" como "prejuicio" para establecer taxonomías sociales y meritocracias que no hacen sino justificar la desigualdad humana.

\section{ABSTRACT}

The present paper tries to study some of the basic rudiments that contributed to the change of paradigm about mental deficiency produced in the first decades of the $\mathrm{XX}^{\text {th }}$ century. Taking as main sources the Binet and Simon's contributions from France, and the Sante de Sanctis' from Italy, it's analysed the construction of the category "abnormal childhood", relating it to the new and privileged space of observation that supposed the Obligatory School.

It concludes with a reflection about the fallacious use of the "intelligence" as "prejudice" in order to establish social taxonomies and meritocracies that they don't make but to justify the human inequality.

A comienzos del presente siglo, el italiano Sante de Sanctis (1862-1935), describió una nueva entidad nosológica al intentar establecer el diagnóstico diferencial de la deficiencia mental con un cuadro psíquico en el que los niños: "escapan al diagnóstico de idiocia o de imbecilidad y más bien se les debe considerar bajo el concep-

\footnotetext{
* Trabajo realizado en el marco del Proyecto de Investigación n PB-94-0060, subvencionado por la DGICYT. 


\section{RAFAEL HUERTAS Y MERCEDES DEL CURA}

to de demencia precoz, en el sentido de que presentan síntomas de esta psicosis, tales como buena memoria, buena capacidad de percepción, cosas todas que contrastan con una inestabilidad extrema de la atención, una debilidad o ausencia de formación del pensamiento de orden superior, perturbaciones graves de la actividad voluntaria, del carácter y de las actitudes, tales como negativismo, tendencia a las acciones rítmicas, impulsividad..."1.

La influencia de la nosografía Kraepeliniana se deja sentir en esta nueva mirada hacia el niño loco y el término de "demencia precocísima", en clara alusión a la dementia praecox de Kraepelin, es aceptado y asumido por diversos autores ${ }^{2}$, hasta llegar a la definición de esquizofrenia infantil establecida ya en $1937^{3}$.

Pero si para la paidopsiquiatría se abría un vasto campo que fue configurándose en torno al concepto de psicosis infantil, la psico-pedagogía había empezado ya a actualizar su discurso y ampliar sus competencias incorporando los avances de la psicología experimental y, de manera particular, aprovechando el nuevo y privilegiado espacio de observación que le facilitaba la Escuela Obligatoria. Gonzalo Rodríguez Lafora afirma, en este sentido que: "Excepto en los niños anormales profundos, en los cuales la segregación con respecto a los normales la hacen las propias familias, el primero que da la voz de alarma sobre la anormalidad intelectual de un niño es el maestro de la escuela primaria. Fúndase éste de ordinario, para tal diferenciación, en el insuficiente rendimiento escolar del alumno o en su inadaptabilidad a la escuela. Para considerar a un niño sospechoso de retardo intelectual, ha dado Binet como norma el grado de su retraso escolar" 4 .

El objeto del presente ensayo es analizar los principios básicos del cambio de paradigma que sobre la deficiencia mental se produce al comienzo del siglo XX, así como identificar los principales elementos y "prejuicios" que contribuyeron a la construcción del concepto de "infancia anormal".

1 Existen varios trabajos de este autor dedicados a esta nueva entidad y publicados entre 1906 y 1909; la cita textual ha sido tomada de SANCTIS, S (1908), "Dementia praecocissima catatonica oder Katatonie des früheren Kindesalters?", Folia neurobiologica, 2: 9 -12, p.9.

2 Así, AuBry, E. (1910), "Psychoses de l'enfance à forme de démence précoce", L'Encéphale, 10: $272-278$.

3 Tal descripción fue presentada en el Primer Congreso Internacional de Psiquiatría del Niño, celebrado en París en 1937 y publicado con detalle en LUTZ, J. (1937), "Über die Schizophrenie in Kindersalter" Arch. Neurol. Neurochir. Psych., 39: 335-372 y 40: 141-161. El esquema que sigue, aplicado al niño, es muy similar al de Bleuler.

4 LAFORA, G. R. (1917), Los niños mentalmente anormales, Madrid, La Lectura, p. 165. 


\section{LOS NIÑOS ANORMALES}

Alfred Binet (1875-1911), director desde 1894 del Laboratorio de Psicología y Pedagogía de la Sorbona, desarrolló, en efecto, una intensa actividad en las escuelas como consecuencia de la campaña iniciada en 1899 por la Société Libre pour l'Etude Psychologique de l'Enfant, que culminó en 1904 con la creación de una Comisión interministerial encargada de acometer "el estudio de las condiciones en las que debería estar prevista la educación de los niños anormales" 5 .

A. Binet, miembro de la Comisión, inició una serie de trabajos encaminados a establecer científicamente las diferencias antropométricas y mentales entre los niños normales y los anormales con el propósito de facilitar el diseño de programas de instrucción para estos últimos. Junto con su más directo colaborador, Théodore Simon (1873-1961), llevó a cabo una serie de trabajos publicados en 1905 en L'Anné Psychologique 6 , en los que se describía un método para medir la inteligecia basado en la aplicación de una escala de tareas ordenadas por su dificultad creciente de acuerdo con la edad de los niños. Trabajos que se agrupan y culminan en el libro Les enfants anormaux (1907).

En el avant-propos de dicha obra, sus autores se desmarcan claramente de la tradición médico-pedagógica desarrollada en Francia a lo largo del siglo XIX. La alusión a pioneros como Itard, Falret o Voisin era poco menos que obligada pero, aunque "es costumbre citar con respeto los nombres de los precursores", aseguran que "sus esfuerzos no obtuvieron ningún resultado" . La crítica más dura se la dedican al franco americano E. Seguin, opinando que en sus obras hay páginas de interés, pero también muchos puntos oscuros e, incluso, absurdos ${ }^{8}$, y reservan sus elogios para Bourneville, por sus continuas demandas a la administración para que en las escuelas públicas se instaurasen clases especiales para niños anormales.

5 En la "Lettre-Préface a M. Alfred Binet", firmada por el senador y presidente de la Comisión León Bourgeois, y publicada en Binet, A. y Simón, TH. (1907), Les enfants anormaux, París, A. Colin. pp. V-VIII, se explican las razones de su creación y se indica que estuvo formada por "educadores, médicos, hombres de ciencia y representantes de los servicios administrativos interesados", p. VI.

" Binet, A. y SimON, Th. (1905a), "Sur la nécessité d'établir un diagnostic scientifique des états inférieurs de l'intelligence", L'Anné Psychologique, 11: 163-190; (1905b), "Méthode nouvelle pour le diagnostic du niveau intellectuel des anormaux", L'Anné Psychologique, 11: 191-244.; (1905c) "Application des méthodes nouvelles au diagnostic du niveau intellectuel des anormaux d'hospice et d'école primaire", L'Anné Psychologique, 11:245-336.

7 BINET Y SiMON (1907), p. 4.

8 Mencionan la obra de Seguin Traitement moral, hygiène et éducation des idiots et des autres enfants arriérés, aparecido en 1846. 
El intento de ruptura epistemológica con respecto a los alienistas que abordaron el problema de la deficiencia mental en la centuria anterior, explica esta actitud hacia los clásicos decimonónicos. Binet y Simon exponían, en 1905, sus objetivos de la siguiente manera: "Nuestro propósito consiste en poder medir la capacidad intelectual del niño que nos traen a fin de averiguar si es normal o atrasado. Debemos, por tanto, limitarnos solamente a estudiar sus condiciones actuales. No nos interesa su historia pasada o su futuro; en consecuencia dejaremos de un lado su etiología y no intentaremos distinguir entre idiotez congénita y adquirida. Por razones más importantes prescindiremos de toda consideración de anatomía patológica que pueda explicar su deficiencia intelectual. Esto en cuanto a su pasado. En lo concerniente a su futuro, practicaremos la misma abstención. No intentamos establecer o elaborar predicción alguna, dejando sin respuesta la pregunta acerca de si su atraso es superable o acaso susceptible de mejora. Nos limitaremos a averiguar la verdad respecto a su estado mental actual"'.

Afanes cientifistas de objetividad que chocan con la tradición anatomoclínica de buena parte de alienismo francés ${ }^{10}$, pero sobre todo, intentan superar la preocupación por las causas y por el pronóstico, eje de las discusiones conceptuales y metodológicas sobre la deficiencia mental durante todo el siglo anterior ${ }^{11}$. Se trata, si se nos permite la expresión, de una cierta desmedicalización de los niños deficientes con la introducción de unos criterios clasificatorios, ciertamente novedosos, basados en la medición de la inteligencia y en el establecimiento de parámetros, como el "nivel de edad", con los que poder ordenar y normativizar a la población escolar.

Merece la pena destacar que Binet y Simon hablan siempre de "nivel de edad" para clasificar a la mayoría de los niños de un grupo de edad que superen una escala de su test, estableciendo, a partir de ese "nivel de edad", el "nivel mental" de un niño determinado. Concepto dinámico que implica la posibilidad de poder pasar de un "nivel" a otro; no en vano la pretensión final de los autores franceses era identificar niños retrasados para someterlos al nivel académico que les correspondería por su edad. Por el contrario, la expresión "edad mental", acuñada por el alemán Wihelm

9 El texto, publicado originariamente en L'Anné Psychologique, ha sido repetidamente citado; así Gould, S. J. (1984), La falsa medida del hombre, Barcelona, Antoni Bosch, p. 151; LóPEz CEREzo, J.A. y LUJAN, J.L. (1989), El artefacto de la inteligencia, Barcelona, Anthropos, p. 135.

i1) Sobre el papel del método anatomoclínico en el alienismo francés véase PESET, J. L. (1993), La.s heridas de la ciencia, Salamanca, Junta de Castilla y León, pp. 161 y ss. También HuERTAS, R. (en prensa), "L'alienismo e la mentalità anatomoclínica: l'opera di J. E. D. Esquirol".

1 Sobre este particular puede verse LANTERI - LAURA, G. (1972), "La chronicité dans la psychiatrie française moderne", Annales ESC, 27, (3): 548-556; Álvarez, R., HuerTAS, R. y PeSET, J. L. (1993), "Enfermedad mental y sociedad en la segunda mitad del siglo XIX", Asclepio, 45, (2): 41-60. Para Inglaterra, JACYMA, J. S. (1982), "Somatic theories of mind and the interest of medicine in Britain, 18501879”, Medical History, 26: 233-358. 
Stern ${ }^{12}$, sugiere, como bien han indicado J.A. López Cerezo y J.L. Luján, algo "endógeno, fijo y similar a la edad cronológica"13.

De este modo, el cambio de las condiciones y los objetivos del observador y, sobre todo, la ampliación de la población observada, implicó la definición de nuevas categorías ya que el concepto de "infancia anormal" englobó rápidamente no solo a los deficientes mentales, sino también a los niños "inestables", término que Binet y Simon prefieren al de indisciplinados ${ }^{14}$.

Una amplia gama de actitudes y aptitudes tienen cabida, pues, dentro de la noción de anormalidad aplicada a la infancia; así, "cl epíteto niño anormal no responde a un tipo único"15, existiendo una variabilidad de grados de inteligencia entre el "idiota vegetativo" y el "débil superior". De igual modo, recelan del lenguaje administrativo, considerando equívocos términos como "anormal médico" y "anormal pedagógico".

Para los deficientes mentales mantienen los términos idiota, imbécil y débil mental, pero redefiniéndolos y recurriendo a la mayor o menor habilidad en el manejo del lenguaje hablado o escrito como criterio clasificatorio. De esta manera, "Es idiota todo niño que no llega a comunicarse por la palabra con sus semejantes, es decir, que no puede ni expresar verbalmente su pensamiento ni comprender el pensamiento, verbalmente expresado, de los demás, siempre que no haya una alteración de la audición, ni de los órganos fonadores que expliquen esta pseudo-afasia, que es debida enteramente a una deficiencia mental"16.

La imbecilidad se aplica a los niños que "no llegan a comunicarse por escrito con sus semejantes, es decir, que no pueden expresar su pensamiento a través de la escritura, ni leer lo escrito o lo impreso, o más exactamente comprender lo que leen, siempre que ninguna alteración de la visión o alguna parálisis motriz del brazo no expliquen la no adquisición de esta forma de lenguaje, defecto de adquisición que es debido a una deficiencia intelectual"17.

Finalmente, "es débil todo niño que sabe comunicarse con sus semejantes a través de la palabra y la escritura, pero que muestra un retardo de dos o tres años en el curso de sus estudios, sin que este retraso sea debido a una insuficiencia de escolaridad"18.

Como bien puede verse, las definiciones propuestas introducen novedades importantes; por un lado, su íntima relación con las actividades escolares de los niños y la posibilidad de que, efectivamente, sea el maestro el primero en detectar las deficien-

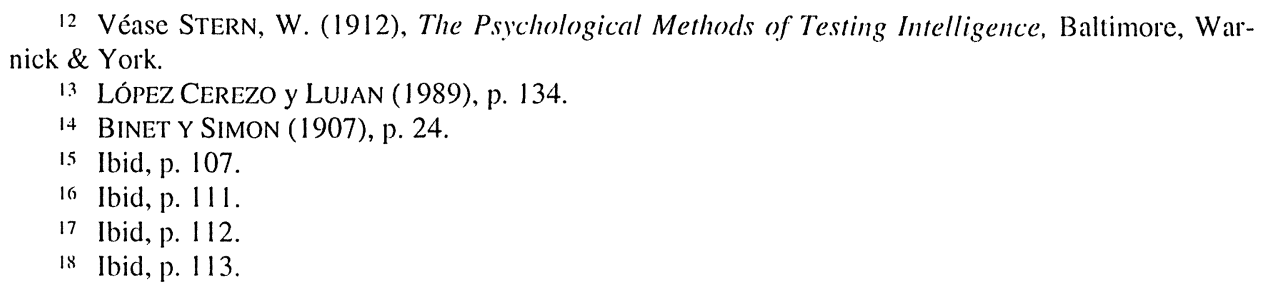

12 Véase Stern, W. (1912), The Psychological Methods of Testing Intelligence, Baltimore, Warnick \& York.

1.3 LÓPEZ CEREzo y LUJAN (1989), p. 134.

14 BINET Y SIMON (1907), p. 24.

15 Ibid, p. 107.

16 Ibid, p. 111

17 Ibid, p. 112.

18 Ibid, p. 113 
cias, sobre todo las menos profundas. Por otro, la concepción psicológica del retraso mental que hace obligatorio descartar alteraciones físicas y/o sensoriales tradicionalmente asociadas a los cuadros de idiocia. Por fin, el intento de superar la evidente dificultad que suponía distinguir la deficiencia mental y el escaso nivel cultural en un momento en el que el analfabetismo de los padres o las dificultades de escolarización entre los hijos de los trabajadores, inquilinos naturales de la Escuela Obligatoria era la norma. De ahí, que se acabe acuñando el término de "falsos anormales" para designar a muchachos sin déficit intelectual que son "simplemente ignorantes"19.

En cualquier caso, el retraso escolar se convierte, desde las primeras décadas del siglo XX en preocupación de médicos y educadores que intentarán encontrar causas "psicológicas" o "bio-psicológicas" a la desadaptación o a las dificultades de aprendizaje de una serie de niños insumisos, inquietos o, simplemente, aburridos ante la normativización escolar, y para los que se esgrimirá el diagnóstico de "anormales", formulación novedosa pero que asimila y actualiza viejos conceptos degeneracionistas aplicados al niño $0^{20}$.

\section{MEDICINA Y PEDAGOGIA}

Aunque son muchos los autores que siguiendo el camino emprendido por Binet y Simon, se ocuparon de la "infancia anormal" en el primer tercio del siglo $\mathrm{XX}^{21}$, citaremos aquí la aportación del italiano Sante de Sanctis porque además de diferenciar, como ya hemos visto, la "demencia precocísima", propone una doble sistematización de la deficiencia mental, en la que incorpora las novedades psico-pedagógicas que el nuevo siglo va produciendo, pero sin renunciar a una tradición médica de arraigo innegable. La influencia de Seguin es clara, pero no lo es menos la de Binet y Simon o la de María Montesori (1870 -1952) ${ }^{22}$.

19 Ibid, p. 114

20) De hecho, en muchas obras sobre "infancia anormal", dicho término viene a sustituir al de "infancia degenerada", sin que se produzcan demasiadas modificaciones en la valoración "moral" de estos niños, VARELA, J. y Álvarez-URIA, F. (1991) Arqueología de la escuela, Madrid., La Piqueta, pp. 216 y ss.

21 Para España, véase, entre otras aportaciones, Pozo, A. (1958), "La educación de deficientes en España: los cincuenta primeros años de su desarrollo (1875-1925)", Revista Española de Pedagogía, 1824.; SCheEREnberger, Sh.O. (1984), Historia del retraso mental, Madrid; Escolano, A. (1997), "Pedagogía y Psicología", En Huertas, R., Romero, A.I. y Álvarez, R. (coords.), Perspectivas psiquiátricas, Madrid, pp. 27-39.

22 Tiene muy en cuenta las propuestas pedagógicas de su compatriota, en particular las de la $2^{\mathrm{a}}$ edición de la obra MONTESORI, M. (1913), Il metodo della pedagogia scientifica applicato all'educazione infantile. 
La clasificación final que de Sanctis ofrece es, como acabamos de indicar, doble (tabla I): una, eminentemente médica, en la que se tienen en cuenta las causas y el correlato clínico - manifestaciones y secuelas- de los pacientes, y, otra de enfoque más pedagógico en la que se tiende a identificar las deficiencias "intelectuales" de los mismos.

En la primera distingue entre frenastenia cerebropática, debida a una enfermedad del cerebro diagnosticable y sobrevenida durante una de las fases del periodo evolutivo intra o extra uterino; frenastenia biopática, en la que no hay evidencias de enfermedades graves del sistema nervioso central y cuyo origen se encuentra en desórdenes de la evolución prenatal del cerebro independientemente de las causas heredadas o congénitas precoces; $y$, finalmente, un tipo de frenastenia mixta en función de su etiología y sintomatología, la biocerebropática, donde incluirá a los disglandulares.

La segunda clasificación, sin embargo, remite a la "anormalidad" entendida como transtorno "moral", considerando una anormalidad intelectual y otra afectiva o de carácter cuyos rasgos fundamentales —aunque no absolutos_ son la "inestabilidad" psíquica y el retraso escolar ${ }^{23}$. En este último grupo incluye a los anormales de conducta o inestables puros, en muchas ocasiones suficientes intelectualmente, entre los que se encontrarían los "inestables amorales o hipomorales", también denominados "inmorales constitucionales", con un fuerte gravamen hereditario y tendencias criminales.

La "anormalidad" aparece en ésta y otras clasificaciones ligada siempre a causas psico - orgánicas, biológicas y mesológicas. Aunque ya Binet lo había apuntado, para de Sanctis las causas sociales revisten especial importancia en la etiología de las deficiencias: "La pobreza, el abandono moral, la mala educación familiar y particularmente las intoxicaciones que los psiquiatras llaman «voluntarias» - principalmente el alcohol- figuran muy a menudo en la etiología de la deficiencia intelectual y moral"24.

Nuestro autor tiene en cuenta la existencia de individuos cuyo retraso mental puede superarse mediante un régimen pedagógico adecuado. Respetando la terminología de Binet y Simon, los denominará "falsos anormales", mostrándose de acuerdo con los autores franceses al señalar que "la fórmula etiológica del falso anormal podría enunciarse así: herencia libre o ligeramente gravada - enfermedades comunes o intoxicaciones en curso o ya sufridas - influencia predominante de factores ambientales (familiares y sociales)"25.

2.3 SANCTIS, S. (1914), Educazioni dei deficienti, Milán, F. Vallardi, pp. 4 y ss.

24 Ibid, p. 93.

25 Ibid. p. 140. 
Al igual que Lafora, tanto Binet como de Sanctis otorgan una extraordinaria importancia al papel del maestro en el despistaje y diagnóstico precoz de las deficiencias mentales en la infancia. Para el psicólogo francés "es anormal intelectual verdadero ( y por tanto seleccionable) el alumno que habiendo pasado los nueve años y habiendo frecuentado regularmente la escuela, tenga un retraso de tres años"26, y para el médico italiano "cuando un alumno se muestra inestable (rebelde, excitable, indisciplinado, violento, impulsivo, con mala conducta) sin tendencia a corregirse, durante los tres primeros meses del año escolar, aún asistiendo siempre a la escuela y teniendo siempre como guía un maestro paciente e inteligente, con gran probabilidad, se le puede declarar inestable, es decir anormal verdadero del carácter"27. No deja de resultar esclarecedor que su categorización venga definida por el éxito o no de las técnicas de educación: el fracaso del especialista acaba convirtiéndose así en criterio clasificatorio de primer orden.

Diferenciaciones clasificatorias cuya artificiosidad es manifiesta pero que permiten establecer etiquetas con gran facilidad: la falta de rendimiento o la inadaptación a las normas escolares que acabaron definiendo la anormalidad infantil, sirvieron también para asimilar ésta a la infancia "viciosa" o delincuente ${ }^{28}$.

La escuela aparece, pues, como un medio regenerador, de educación y de integración moralizadora, destinado a inculcar docilidad y aceptación de las normas burguesas y a erradicar todo germen de conciencia revolucionaria ${ }^{29}$. Para los que pongan dificultades a dicho proceso de "socialización", se diseñarán colegios de corrección pedagógica, reformatorios o unidades de psiquiatría infantil, según se trate de niños maleducados, delincuentes o $\operatorname{locos}^{30}$.

$26 \quad$ Ibid.p. 157.

27 Ibid. p. 164

28 Existe también una amplia bibliografía sobre la medicalización de la infancia delincuente; véase, a modo de ejemplo, Álvarez-Uría, F. (1987), "La infancia tutelada", en HuerTas, R., ROMERO, A.I. y Álvarez, R. (coords.), Perspectivas psiquicitricas, Madrid, CSIC, pp.177-189; Tambićn HuERTAS, R. (1991), El delincuente y su patología, Madrid, CSIC.

29) Véase Pinel, P. y ZaFiropoulos, M. (1978), "La medicalisation de l'échec scolaire. De la pédopsychiatrie à la psychanalise infantile", Actes de la Recherche en Sciences Sociales, 24: 23-49; MUEL, F. (1981), "La escuela obligatoria y la invención de la infancia anormal", En VV.AA., Espacios de Poder, Madrid, La Piqueta, pp. 123-143.; VArelA, J. y Álvarez-Uría, F. (1991), pp. 209 y ss.

31) Véase ÁlvareZ-Uría (1987), p.184. Sobre el acercamiento médico al niño a comienzos de este siglo analizado de una manera integral y no solo desde el punto de vista del control social, podrá verse un magnífico trabajo de BALLESTER, R. y BALAGUER, E. (1995), "La infancia como valor y como problema en las luchas sanitarias de principio de siglo", Dynamis, 15, pp. 177-192. 


\section{LA NORMA Y SUS EXPERTOS}

No obstante, las nuevas instituciones, el desarrollo de técnicas, el concurso de nuevos expertos (psicólogos, pedagogos, etc.) o la ampliación paulatina de la actuación paidopsiquiatrica a ámbitos diversos (jurídico-criminológico, social, pedagógico, etc.), no parecen haber aportado novedades nosográficas en los últimos tiempos. El mundo de la oligofrenia, por un lado, y el de las psicosis infantiles, por otro, siguen siendo los más claramente definidos, a pesar del "ruido accesorio" provocado por lo que Otta de Leonardis ha llamado "mito de la competencia especializada", mediante el que pretende explicar cómo en la labor de los especialistas se concreta el paradigma racionalista problema-solución, de manera que "a una definición racional, científica, codificada, del problema corresponde una solución técnica racional"31. La solución institucional determina, en no pocos casos, la forma en que se analiza e, incluso, se formula el problema, hasta el extremo de prescindir o ignorar aquéllos que no entran dentro de los límites de saberes y estrategias que ese marco institucional es capaz de desarrollar. La llamada "infancia anormal" puede ser un ejemplo suficientemente elocuente, pero puede haber muchos más, así la medicalización de las conductas "antisociales", o la definición de "Paciente Crónico Adulto Joven", con la que, recientemente, se ha pretendido caracterizar a una serie de individuos con dificultades en su funcionamiento social que tienen como principal característica "su tendencia a usar inadecuadamente los servicios de Salud Mental de forma tal que consumen gran cantidad de energía y tiempo de los clínicos sin que sea posible establecer planes viables de tratamiento"32 .

Como puede verse, las dificultades para aislar "especies morbosas" con las que elaborar nosografías más o menos complejas son manifiestas. Los prejuicios sociales e institucionales matizan, cuando no determinan directamente, los juicios clínicos "objetivos", basados, a su vez, en prejuicios más o menos científicos. Estas difícultades las tuvo, desde su comienzo, el acercamiento médico-pedagógico a la defïciencia

31 LEONARDIS, O. (1992), "Políticas sociales: reinventar nuevos parámetros", En Ál.VAREZ-URíA, F. (coord.), Marginación e inserción. Los nuevos retos de las políticas sociales, Madrid, pp. 55-66, p. 56.

${ }^{32} \mathrm{La}$ formulación primigenia del concepto se encuentra en PEPPER, B. y RYGLEWICZ, H. (cds.) (1982), The Young Adult Chronic Patients, San Francisco. Las críticas a esta categoría diagnóstica no se hicieron esperar, véase ESTROFF, S.E. (1987), "No more Young Adult Chronic Patient", Hospital Community Psychiatry, 38: 5. Una de las primeras revisiones que se hicieron en España sobre el tema fue la realizada por DESviat, M., FERnÁNDEZ-LiRIA, A. y RENDueles, G. (1986), "La nucva cronicidad", En EsPinosA, J. (coord.), Cronicidad en Psiquiatría, Madrid. Véase también FERnÁndEZ-Liria, A. (1991), El concepto de Paciente Crónico Adulto Joven: Pertinencia y relevancia para la planificación y organización de los servicios de Salud mental, Madrid, Tesis doctoral inédita. Una crítica a cste concepto en el contexto de un análisis más amplio, puede verse en HUERTAS, R. (1993), "El mito del 'bienestar' y la política social", Utopías, n 154 , pp. 18-33. 
mental. Dicho acercamiento —iniciado por Itard a comienzos del siglo pasado — con sus posteriores desarrollos, constituye, en nuestra opinión, un caso particular, pero muy significativo, en el que pueden evaluarse las razones, pero sobre todo las consecuencias, de un sistema clasificatorio basado en criterios diferentes a los comúnmente aceptados por las Ciencias Naturales a las que, precisamente, la Medicina pretendía coronar.

\section{A MODO DE EPÍLOGO: INTELIGENCIA Y DESIGUALDAD}

Una última reflexión sobre la clasificación de los seres humanos por su inteligencia parece obligada. Por un lado, los trabajos de Binet y Simon han sido criticados por algunos autores que ven en ellos el germen de una ideología segregacionista destinada a eliminar del "circuito escolar" a los más torpes ${ }^{33}$. Como ya hemos indicado, su intención fue justo la contraria, propiciar la recuperación de los niños más atrasados; en este mismo sentido Stephen J. Gould opina que Binet no consideró el Cociente Intelectual como un recurso para clasificar jerárquicamente a los alumnos de acuerdo con sus valores intelectuales sino que elaboró su escala con fines muy limitados: el encargo que le había hecho el Ministerio de Educación de idear una guía práctica para detectar a aquellos niños cuyos pobres resultados escolares indicaban su necesidad de recibir educación especial ${ }^{34}$.

No por ello puede negarse que, a pesar de sus buenas intenciones, sus aportaciones ofrecieron al darwinismo social una eficaz herramienta con la que medir la desigualdad intelectual de los seres humanos. La estratificación del "reparto de tareas" en una sociedad de clases quedaba consagrada en virtud de la diversidad intelectual de la especie humana. El discurso se completó hábilmente con las teorías hereditarias de la inteligencia, presentes desde el comienzo del movimiento eugénico ${ }^{35}$ y el determinismo biologicista del llamado Cociente Intelectual.

En 1969, Arthur R. Jensen publicó un extenso e influyente artículo en el que actualizó el discurso de la determinación biológica de la inteligencia ${ }^{36}$. Dicho trabajo no era sino el inicio de una ofensiva que pretendía negar, una vez más, las condicio-

33 Véase TORT, M. (1974), Le Quotien Intellectuel, París, Maspero, pp. 184 y ss. También SANDro, M. (1974), "Le 'test d'intelligence' est une infamie", Journal Témoignage Chrétien (2-mayo), pp. 27-28; (18-julio), pp. 16-17.

34 Gould, (1984), p.151.

35 Sobre los orígenes del discurso eugénico véase ÁlvareZ, R.(1985), Sir Francis Galton, padre de la eugenesia, Madrid, CSIC.

36 Recogido en JENSEN, A.R. (1972), Genetics \& Education, Londres, Methuen. El original de 1969 llevó por título "How Much Can We Boost IQ and Scholastic Achievement?" y fue publicado en la Harvard Educational Review. 
nes sociales en el análisis del comportamiento humano, poniendo al día viejos reduccionismos biologicistas y somaticistas. Pocos años más tarde vió la luz el famoso libro de Edward O. Wilson que pretendió explicar, desde la denominada sociobiología, la influencia determinante de la herencia biológica — de lo genético- en los comportamientos sociales ${ }^{37}$.

Nótese que estas ideas surgen en la prestigiosa y conservadora universidad de Harvard, precisamente en el inicio de una etapa política y económica en la que la exigencia liberal del individualismo y del darwinismo social necesitaba apoyos teóricos que se presentaran como incuestionables.

Se irán retomando, de esta manera, taxonomías sociales basadas en las capacidades intelectuales —entendidas como facultades naturales-, en virtud de las cuales se establecerá el lugar que cada uno debe ocupar en las relaciones de producción. Desde profesionales liberales -inteligentes, triunfadores y cualificados- hasta obreros no especializados, la gama de posibilidades es amplia y permitió a no pocos autores relacionar inteligencia con clase social; todo lo cual, junto al papel del "especialista" capaz de colocar al sujeto en el lugar más adecuado dentro de la sociedad, nos coloca a un paso del mundo feliz que Huxley inventó. Pero además, aunque estos planteamientos suponen un evidente refuerzo del neodarwinismo social, se introduce un importante matiz que viene a afianzar todavía más los argumentos de la llamada Nueva Derecha: si el darwinismo social propugna la supervivencia de los más aptos, ahora se puede también explicar "científicamente" el fracaso de los más débiles ${ }^{38}$.

Voces diversas se han alzado contra estos argumentos. Stephen Gould, aunque se podrían citar otros muchos ejemplos ${ }^{39}$, ha denunciado el evidente error metodológico que supone el hecho de que los test de Cociente Intelectual midan una variable independiente - la inteligencia- y el no menos evidente prejuicio de clasificar y ordenar a los miembros de una población en función del grado de inteligencia que posean, sin tener en cuenta la innegable influencia de los factores ambientales, sociales, etc. ${ }^{40}$.

A modo de breve conclusión, nos parece imprescindible insistir en la necesidad de denunciar, desde la Historia y la Filosofía de la Ciencia, no solo los prejuicios de la sistemática, sino la utilización acrítica, cuando no perversa, de los criterios de ordenación de la naturaleza. En el caso que nos ocupa, es evidente que las concep-

37 WiLSON, E.O. (1975), Sociobiology. The New Synthesis, Harvard University Press.

38 Véase LóPEZ CEREZO y LUJAN (1989), p. 146.

39) En nuesto medio, podemos destacar el libro de LÓPEZ CEREZO, J.A. y LUJAN, J.L. (1989), El artefacto de la inteligencia. Una reflexión crítica sobre el determinismo biológico de la inteligencia, Barcelona, Anthropos. Además, en el capítulo 4, titulado "Una filosofía crítica de la ciencia: El caso de la teoría hereditarista de la inteligencia" (pp. 185 y ss.), se ofrece una amplia revisión de lo que es el movimiento de contestación al determinismo biológico en este campo. Remitimos, pues, a la bibliografía citada por estos autores.

40) Véase GouLd, S. (1984), La falsa medida del hombre, Barcelona. 
ciones biologicistas e individualistas olvidan deliberadamente que las propiedades biológicas del ser humano, sus potencialidades genéticas, no se manifiestan directamente, sino que se ven modificadas profundamente por los factores sociales. Los animales heredan, a través de un código genético rígido, las capacidades necesarias para adaptarse al ambiente mediante funciones muy especializadas y experiencias específicas que permanecen invariables a lo largo de la vida. Por el contrario, la desespecialización y la amplia variabilidad de las condiciones vitales humanas constituye una de las premisas que le permitió dar el paso del mundo de la naturaleza al mundo de la cultura, entendiendo ésta como "naturaleza transformada". Dicho de otro modo, la ausencia de hábitos heredados innatos consolidados en el genotipo humano coloca en primer plano la experiencia individual - la capacidad de aprendizaje- y posibilita una amplia gama de interacciones con el medio ${ }^{41}$. En este marco, la mediatización de lo biológico por lo social condiciona la correlación de las propiedades heredadas y las adquiridas en el proceso de desarrollo individual, en el que la capacidad intelectual es, tan solo, un elemento más. Elemento que, sin entrar en conflicto con la diversidad biológica y cultural del ser humano, no puede ser utilizado como argumento de persecución, segregación o explotación del hombre por el hombre.

41 Sobre la interacción entre lo biológico y lo social tanto en la condición humana como cn el proceso salud-enfermedad, puede verse HUERTAS, R. (1996), "Las ciencias de la salud y el marxismo: sobre la construcción de una teoría de lo social en medicina", Papeles de la FIM, 5 (2 ápoca): 35-44. 


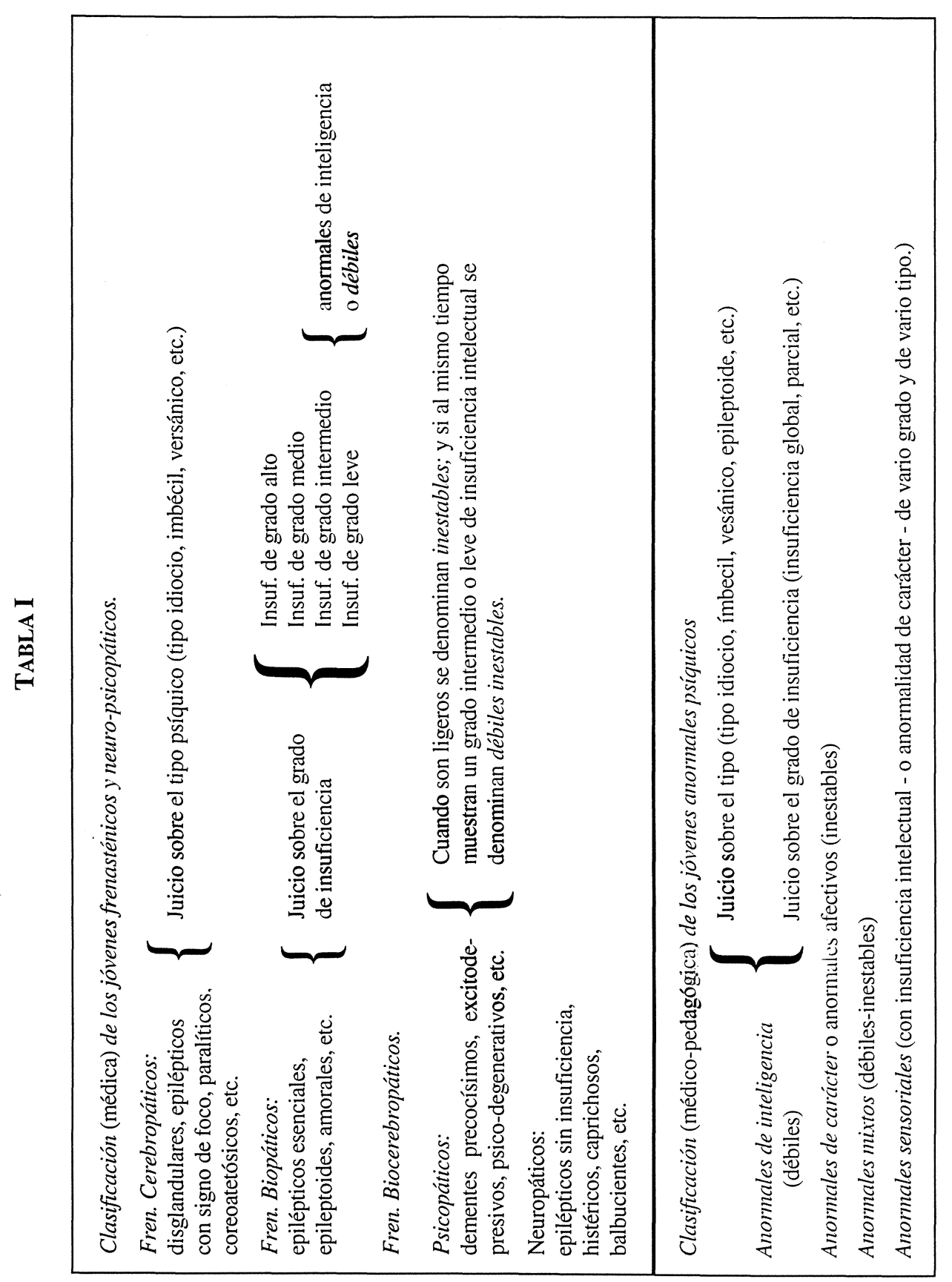

Asclepio-Vol. XLVIII-2-1996 